\title{
Older Adults' Explanatory Models of High Blood Pressure
}

\author{
Margo-Lea Hurwicz, Margaret Rose \\ Department of Sociology, Gerontology \& Gender, University of Missouri-St. Louis, St. Louis, MO, USA \\ Email: Hurwicz@umsl.edu
}

Received 29 March 2016; accepted 15 April 2016; published 27 May 2016

Copyright (C) 2016 by authors and Scientific Research Publishing Inc.

This work is licensed under the Creative Commons Attribution International License (CC BY). http://creativecommons.org/licenses/by/4.0/

(c) (i) Open Access

\section{Abstract}

Shared Explanatory Models (EM) of High Blood Pressure (HBP)/Hypertension (HTN) were explored using systematic data collection and analysis methods from cognitive anthropology. Older adults who were members of a Medicare HMO in Los Angeles were asked to list all the illnesses experienced by older adults that they could recall, and those listing HBP or HTN were asked to further list and discuss its symptoms, causes, treatments and prevention. Responses were tape recorded, transcribed, and analyzed to develop a systematic "sentence completion by card sort" follow-up procedure. Consensus Analysis (CA) of the systematically collected data identified shared EM for HBP/HTN. The model presented here is similar to models of HBP/HTN described by researchers working with patients from different regions and different ethnic groups, suggesting that there is a widely shared lay or popular model for this disease. Stress, lifestyle (diet, exercise, weight, and substance use), heredity and aging are thought to be the major causes of HBP/HTN. Physicians are thought to be the appropriate source of care, as HTN/HBP is serious, life threatening, and potentially disabling. The study of cultural understandings and shared EM of disease has direct relevance for clinical practice and public health education. For a disease such as HTN/HBP, knowing where and how such explanations differ systematically between patients and clinicians, and what impact this may have on patterns of adherence to prescribed treatment is a crucial area of concern.

\section{Keywords}

Older Adults, Hypertension, High Blood Pressure, Explanatory Models, Consensus Analysis

\section{Introduction}

Cultural beliefs and values shape the way disease and illness are understood and treated in any given society. This is true for illness/disease in general, for specific types of illness/disease and for particular episodes of ill- 
ness experienced by individuals. Therefore, the study of cultural influences on help-seeking for health problems and adherence to treatment regimens has direct relevance for both clinical practice and public health efforts.

Kleinman's [1] [2] Explanatory Model framework has had a major influence on anthropological studies that focus on how culture shapes health-related beliefs and behaviors, especially studies of specific disease/illnesses, such as Good's [3] discussion of heart distress in Iran; Helman's [4] examination of EM for colds and fever in suburban London; Blumhagen's [5] [6], Garro's [7] and Heurtin-Roberts' [8] work on hypertension; Baer et al.'s [9] [10] analyses of colds among geographically dispersed Latin Americans; Schrauf and Iris's [11] study of Alzheimer's disease in Chicago, and Hurwicz and Rose's [12] comparison of EM for colds and flu in Los Angeles.

In Kleinman's theoretical framework, EM are defined as ideas "about an episode of sickness and its treatment that are employed by all those engaged in the clinical process," including explanations of the cause, symptoms, treatment and prognosis of the illness [2]. They draw on cultural beliefs about health and sickness that are widely shared by members of a given society or social group. EM are "formed and employed to cope with a specific health problem" experienced by an individual and responded to by that individual, their family and friends and health practitioners in an attempt to resolve or alleviate that health problem. They are specific to individual illness episodes and incorporate elements of the individual's own experience and situation along with widely shared health beliefs. Therefore, EM do not directly correspond to culturally shared beliefs.

Health practitioner and patient EM often differ. In fact, Helman states that "Consultations with a doctor are actually transactions between lay and medical EM of a particular illness" [13]. Such transactions affect the success of any clinical encounter with regard to treatment and outcome. Therefore, knowing where and how patient and clinician EM differ and what impact this may have on a patient's adherence to prescribed treatment is a crucial area of concern. It is also an area in which cultural studies of illness perceptions make relevant contributions to health education, patient care and successful health outcomes.

Hypertension (HTN) is a chronic disease with potentially grave consequences when left untreated. As the disease usually does not manifest symptoms, individuals typically are not aware they have this condition until a doctor diagnoses it. Since High Blood Pressure (HBP) is a risk factor for heart attacks and strokes, which can cause paralysis, permanent disability and death, it requires regular management and frequent encounters with health professionals for monitoring. Thus, patients and clinicians must engage in regular, long-term transactions of the kind described by Helman [13] in which the EM of both parties to the clinical encounter come into play.

\section{Background}

The research reported in this paper was influenced by 3 anthropological studies that examined the cognitive domain of HTN or HBP. Using a semi-structured questionnaire based on Kleinman's theoretical framework, Blumhagen [5] [6] explored the perceptions of 103 patients attending a HTN clinic at the Seattle Veterans Administration Medical Center. Selecting elements that appeared in the EM of $20 \%$ of the interviewees, Blumhagen identified a shared EM. He also considered the impact of biomedical information obtained at the clinic on his informants' perceptions.

Blumhagen's informants' model of HTN is a stress model. They recognized 3 major kinds of stress: chronic external stress, chronic internal stress and acute stress. Chronic external stress included single episodes of extreme stress, job stresses and the buildup of normal stresses that come with tension, anxiety and lack of rest. Chronic internal stress included psychological problems and family or interpersonal problems. Acute stress was perceived as any situation that temporarily affected an individual and made that person more vulnerable to becoming "hyper-tense" [5].

Informants saw these causal factors as resulting in what Blumhagen labeled "Hyper-Tension," which is "a state characterized by the experience of nervousness, fear, anxiety, worry, anger, upset, tenseness, over-activity, exhaustion and/or excitement."Although psychosocial factors produced this state by making persons more vulnerable to becoming "hyper-tense," "Hyper-Tension" itself was seen to be a physical rather than psychological condition, involving "a physical change in the person.” Many of Blumhagen's informants also identified alternate causes of "Hyper-Tension," including diet, alcohol, smoking, heredity, and personality traits. Causal factors related to diet included the ingestion of salt, water and fatty foods and overeating in general, which led to being overweight.

As symptoms of "Hyper-Tension,” Blumhagen's informants identified tiredness, dizziness, headache, flushing, anxiety/worry, tension, irritability, losing sleep, and feeling upset or stressed. They saw many of the emo- 
tional factors that cause "Hyper-Tension" as symptomatic of it as well; thus causes and exacerbation were seen as intertwined. They also recognized "Hyper-Tension" as a condition that could result in a number of serious health consequences, including strokes, heart attacks and other vascular problems, paralysis and death.

Whether Blumhagen's informants treated their condition by reducing stress or in some other way associated with their EM, they also attended the HTN clinic for biomedical treatment. Blumhagen identified the two nurse practitioners who dealt directly with patients and the informational pamphlets they provided as the two sources for the biomedical EM in this particular clinic. In general, the biomedical model presented at the clinic was very different from the shared model for "Hyper-Tension." The fact that informants maintained their own model despite exposure to the clinic suggests that "social or psychological forces must exist which require a model of illness based on social stress - otherwise such a 'survival' would rapidly be overcome by the sheer weight of Biomedical opinion.”

Heurtin-Roberts [8] also used Kleinman's EM framework, aggregating individual EM for HBP reported by 60 African-American women who were patients at either a general medicine clinic or a smaller HTN clinic in New Orleans. Her goal was to identify a cultural model shared by the group. She identified two such models, one for "high blood" and the other for "high-pertension.” In describing these two discrete but related models, HeurtinRoberts emphasized the differences between them. One model is of a dietary disease and the other is of a stress-related disorder.

Informants perceived "high blood" as a physical condition in which "thick" or "hot" blood rises "up in the body, clogs, and remains there." The "elevated" blood can remain so for several months. Diet was considered to be the most important cause of "high blood," particularly the consumption of fresh or salted pork, "grease," "seasoning" and salt. "High blood" could also be caused or exacerbated by heredity and by hot weather or high body temperature that caused blood to become hotter and thicker. Stress was additionally identified as a cause of "high blood," although "nerves," anger and worries were not seen to be important factors for this disorder. "High blood" is not curable but can be treated, most importantly by biomedicine and its prescription drugs for HTN. Control of diet was also considered important by Heurtin-Roberts' informants, as was losing weight to a lesser degree. Home remedies for cooling and thinning the blood and lowering its elevation in the body included lemon, vinegar and garlic, but such remedies were perceived to supplement biomedical treatment, not to replace it.

In contrast, "high-pertension" was perceived by Heurtin-Roberts' informants as a disorder related to "bad nerves," stress, worry and intense emotion, such as anger. A person's basic temperament was also considered causal in relation to these factors. "High-pertension" stems "from an excitable, emotional self interacting with one's social milieu." It is episodic and unpredictable because it occurs during moments of intense emotion that cannot always be foreseen. Intense emotion causes the blood to rise suddenly and very rapidly to the head, higher than blood rises in "high blood" and much faster. The blood is believed to rise so high and so suddenly that "high-pertension" is considered to be more dangerous than "high blood" and to be a condition that can cause instant death. After these intense emotions have subsided, however, an individual's blood returns to a lower, and safer, position in the body.

Consistent with this perception of "high-pertension," controlling diet and weight were not seen to be effective strategies for managing it. Prescription medication also was not effective, unless the medication was for relaxing someone or inducing sleep. "Most important for high-pertension was that the individual avoid worrying, relax, rest, stay quiet, and get away from people.” People with "high-pertension” recognized the potentially serious health consequences of their condition and, in fact, used these potential consequences as a threat to control the unacceptable behavior of people in their social environment.

Both Blumhagen and Heurtin-Roberts constructed composite models shared by their informants, thereby moving beyond the particularistic nature of Kleinman's original EM formulation. Nonetheless, both used a methodology that follows Kleinman's primary emphasis on EM as individualized rather than culturally shared. They began by eliciting EM from each of their informants and then employed these as unitary building blocks to create cumulative or frequency models of culture.

In contrast, Garro's work [7] on cultural perceptions of HBP represents a methodological breakthrough that builds on the EM formulation. While beginning from an EM premise and collecting individual EM, Garro included a complementary strategy in her research design that allowed her to collect systematic data from all informants and subject these data to statistically powerful consensus analysis (CA), a method ideally suited to identifying shared cultural knowledge using small samples. This in turn allowed her to make more compelling assertions about which perceptions were shared by her informants and to explain deviations of individual EM from the shared model. 
Like Blumhagen and Heurtin-Roberts, Garro was concerned with developing a cultural model of HBP. She examined consensus and variation in knowledge about HBP/HTN among 21 women and 5 men living in an Ojibway reserve community in Canada. She analyzed data from open-ended interviews based on Kleinman's EM interview format and the results from a true-false questionnaire, finding strong consensus in knowledge about HTN. Garro notes that the strength of her true-false questions is that they generate systematically analyzable data. These data can also "be contrasted with similar data collected from other individuals, such as physicians and other providers of health care" and eventually with data collected from other settings.

The causes of HBP most frequently mentioned by Garro's Ojibway informants were worrying too much, alcohol, overexertion, heredity, being overweight, diet and pregnancy. Thus Garro's informants also emphasized stress as a cause of HBP, while presenting diet and heredity as alternate causes. They perceived HBP "as an episodic illness that comes and goes" with different causes bringing on different illness episodes. Consistent with this view, they might see a particularly stressful situation as initially causing it, with later episodes caused by more chronic stresses.

The concept of balance is a central feature in Ojibway beliefs about illness. Although emotional stress and worrying are major sources of HBP-related imbalance in the body, this imbalance can also be caused by too much of other things, such as too much salt or being overweight. Thus despite the emotional origin of the imbalance caused by stress, HBP was perceived by Garro's informants as a physical disorder rather than a psychological one.

In contrast to the biomedical model, which defines HTN as a chronic condition with no symptoms, Garro's informants saw episodic rises in blood pressure as accompanied by perceptible symptoms, including headaches, dizziness and feeling tired or weak. Treatment was seen as a matter of regulating symptoms. When symptoms disappeared, some informants saw themselves as no longer suffering from HBP/HTN and stopped taking their medication until symptoms returned. Others took their medication regularly, but increased their dosage when symptoms developed. That is, some informants saw their illness as an acute one, while others saw it more as an episodic chronic illness.

Garro's informants also recognized HBP/HTN as a disorder that could have very serious health consequences, including death, strokes, black outs, heart problems and diabetes. She concluded that there is a cultural model of HBP shared by her Ojibway informants. It is "learned from a variety of sources," "provides the basic framework for interpreting perceived symptoms and their causes" and "gives meaning to many of the experiences and actions associated with this illness," despite variation in individual EM.

Taking into consideration the different elements that can contribute to the production of cultural models of illness, Garro proposes that such cultural models be contrasted across diverse samples by using a research design that asks "systematic questions with comparable responses, like the true-false statements." These responses can then "be used to map similarities and differences in distinct cultural settings more precisely."

The present study follows this recommendation, using both quantitative and qualitative methods to discover the cultural knowledge of HBP/HTN shared by Medicare-eligible older adults in Los Angeles, some of whom had been diagnosed with HTN and some of whom had not. As suggested, it uses systematic data collection techniques [14] and consensus analysis [15] [16] to identify shared EM. It further analyzes participants' transcribed narratives for common themes explaining causes, preventions, treatments and symptoms.

\section{Methods}

The analysis presented in this paper is based on data from a study previously described in our paper, Older Adults' Explanatory Models of Colds and Flu [12]:

"Sampling and recruitment were consistent with other research that uses consensus analysis (CA) to identify shared cultural models of illness [9] [11]. Tables providing sample size and validity estimates for different level of agreement [16] [17] were used to determine the target sample size. Thirty participants were randomly selected for exploratory in-depth interviews about late life illnesses from a pool of volunteers who were members of an HMO in Los Angeles. A free listing procedure required them to recall illnesses or health conditions from memory. Free lists of causes, preventions, treatments and symptoms were elicited for each illness mentioned, and coded as present or absent. Matrices of illnesses and their attributes were analyzed to discover common themes. From their accompanying narratives, which had been recorded and transcribed, a set of sentence frames was abstracted, revised and administered to 28 of the 30 participants using a card sorting procedure that was developed for this research [14] (see Table 1 for sentence frames). The objective was to identify shared cultural knowledge about each of the 30 illnesses/conditions men- 
tioned by more than $20 \%$ of the sample."

In the present paper, however, we focus on the part of the data matrix that includes variables measuring cultural knowledge, or the elements of Explanatory Models (EM) for HBP or HTN.

\subsection{Sample}

Nineteen (63\%) of the 30 free-listing phase participants mentioned either Hypertension (HTN) or High Blood Pressure (HBP). Eighteen (60\%) were women and $12(40 \%)$ were men, with a mean age of 78 years (range 70 95). Fifteen (50\%) were widowed, 12 (40\%) were married, one was divorced, one separated, and one never married. Over half ( 18 or $60 \%$ ) had been to college and most of them (26 or $87 \%$ ) described their work status as retired. Thirteen (43\%) identified their religion as Protestant, 9 (30\%) as Catholic, and 6 (20\%) as Jewish. They are largely of European ancestry (23 or 77\%): Within this category 33\% categorized themselves as "American" and not part of any ethnic group. Six (20\%) percent identified their ethnicity as Jewish, 3 (10\%) said they were Italian-Americans, 2 (7\%) said they were Irish-Americans, and one each said they were of German or English descent. The remainder identified as African-American (4 or 13\%) or Hispanic-American (3 or 10\%).

The majority rated their health as good or excellent (22 or $73 \%)$ and $8(27 \%)$ rated their health as fair. None rated their health as poor. However, 19 (63\%) reported one or more chronic conditions, with 15 (50\%) specifically reporting a diagnosis of hypertension. Twenty-nine (97\%) had a regular doctor and all had visited a physician in the past year.

\subsection{Procedures ${ }^{1}$}

Volunteers were telephoned and the study described. If they wished to participate, an appointment was set up for the first author to visit them in their home at a convenient time. During the visit, which lasted between one and 2

Table 1. Consensus analysis of sentence completion/card sort responses for high blood pressure or hypertension and estimated answer key $(\mathrm{N}=28)$.

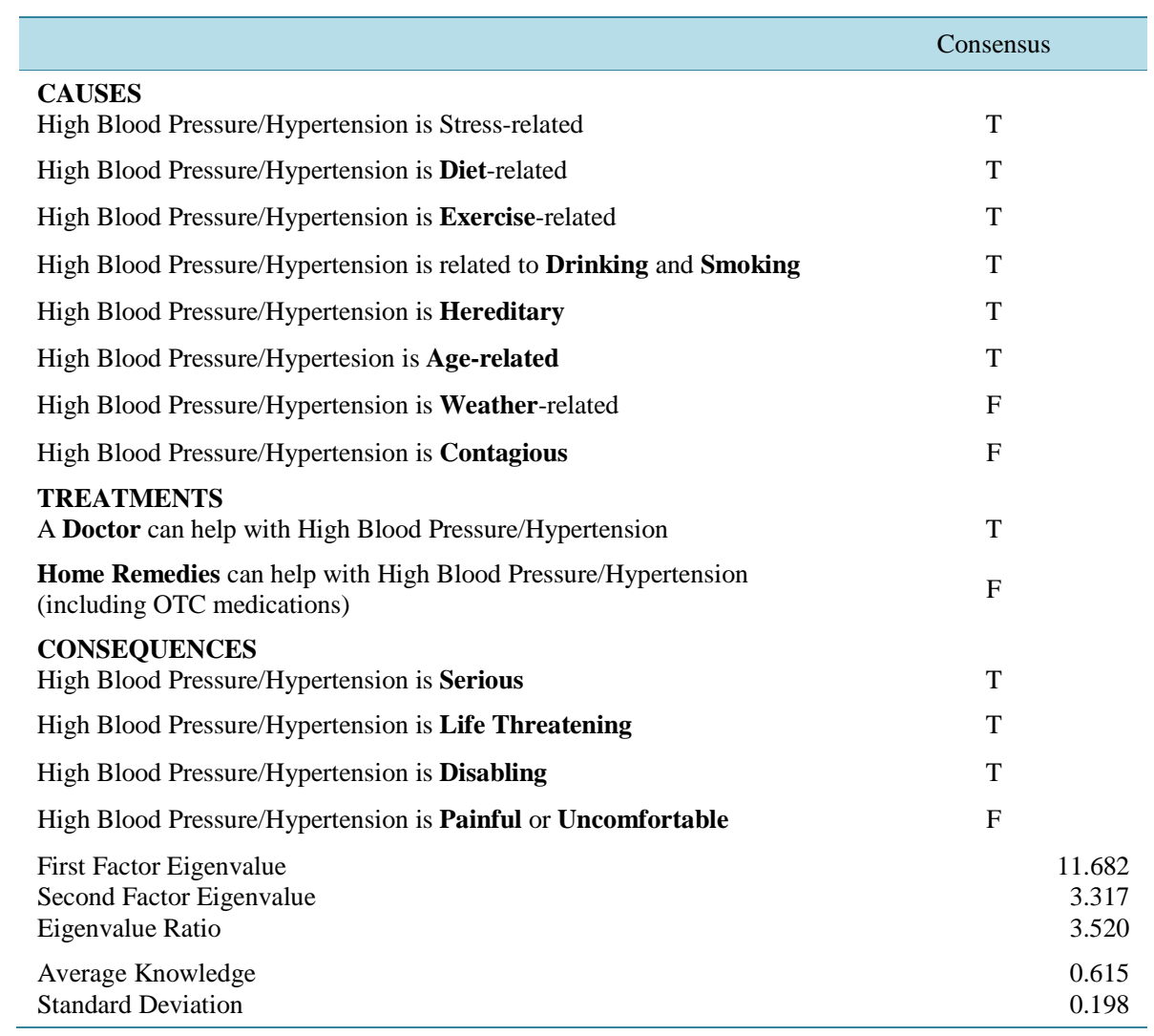

${ }^{1}$ Procedures were reviewed and approved by the Institutional Review Board of the University of Missouri-St. Louis. 
hours, they were asked to list all the health problems/conditions that an adult might face in later life. The 19 participants who spontaneously mentioned HBP or HTN were asked to describe it in more detail (see Table 2). At this point the request for information was normative. They were not asked about their own experience with high blood pressure or hypertension, but rather about their general (cultural) understandings of this illness/disease. The free list responses were recorded, coded and entered into ANTHROPAC [15], a software package designed for the systematic analysis of cognitive anthropology data [12]. In the course of listing attributes of HTN or HBP, the participants also described the reasons for their answers; the tape-recorded narratives were transcribed verbatim.

During a second home visit, 28 of the participants were asked to respond to a more systematic protocol, consisting of 14 sentence frames that had been developed using language from the narratives. As detailed in our previous paper [12]:

"Each sentence includes a blank into which any illness/condition name could be inserted to make it true or false. Each participant was given a set of 30 cards with the names of the most frequently mentioned illnesses/conditions printed on them. For each sentence, they were instructed to sort the cards into a pile that would make the sentence true, and a pile that would make the sentence false. For the example, "(Blank) is life threatening," cancer would most likely make the sentence true; arthritis would most likely make the sentence false. They did not need to recall from memory either the illnesses or the elements of the cultural explanatory models for the illnesses. This method efficiently produced an answer of true or false to 420 (14 $\times 30$ ) sentence/questions for each participant. These data also were entered into ANTHROPAC [15].”

Table 2. Distribution of Responses from Free List Procedure for High Blood Pressure or Hypertension $(\mathrm{N}=19)$.

\begin{tabular}{lr}
\hline CAUSES & Number Recalling (\%) \\
Stress & $10(53)$ \\
Diet & $8(42)$ \\
Overexertion & $6(32)$ \\
Smoking & $3(16)$ \\
Heredity & $3(16)$ \\
Aging & $1(5)$ \\
PREVENTIONS & \\
Improve Diet & $10(53)$ \\
Reduce Stress & $5(26)$ \\
Increase Exercise & $4(21)$ \\
Do Not Smoke & $2(11)$ \\
Take Medication & $1(5)$ \\
TREATMENTS & $18(95)$ \\
Doctor-recommended Medication & $8(42)$ \\
Change Diet & $4(21)$ \\
Exercise & $1(5)$ \\
Rest & $1(5)$ \\
Quit Smoking & $1(5)$ \\
Over-the-counter Medication & $5(26)$ \\
SYMPTOMS & $4(21)$ \\
No Symtoms & $3(16)$ \\
Hizziness & $3(16)$ \\
Otheadache Pain (Specified) & \\
\hline & \\
\hline & \\
\hline
\end{tabular}


The participant $(\mathrm{N}=28)$ by question $(\mathrm{N}=14)$ matrix for the "Hypertension/High Blood Pressure" card was used in the following analyses.

\subsection{Operational Hypotheses}

A priori hypotheses, based on literature review and the transcripts of the narratives, were that agreed-upon causes/exacerbating factors for HTN/HBP would include stress, other lifestyle variables (diet, exercise and weight, drinking and smoking), and maybe heredity, but would not include contagion and weather. There would be agreement that HTN/HBP is serious and life-threatening, but not painful, and that a medical doctor could help. Hypotheses about age as a cause, and disability as a consequence also were considered, but expected results for these variables were not specified.

\subsection{Data Analysis}

The analysis plan for the HBP/HTN data was the same as the plan for the colds and flu data [12]:

"The sentence frame/card sort data were analyzed using the Consensus Analysis procedure in ANTHROPAC [15]. This procedure analyzes the person-by-question data matrix to provide an estimate of each individual's level of cultural knowledge about a given domain. The statistical model is based on the assumption that the questions tap a shared cultural domain, and assesses whether this assumption is met. If it is met, the estimates may be used to develop a key to the culturally agreed upon answers to the questions. Two additional assumptions are that the participants answer the questions without consulting one another, and that all of the questions are equally representative of the cultural domain under consideration.

A more complete description of the analytic procedures is available elsewhere [15] [16]; however, the basic elements of the statistical analysis are presented here. A correlation matrix representing agreement among the responses of the participants is factor analyzed after a mathematical correction for guessing, and the factor loading for each participant is taken to be their level of cultural knowledge or expertise. For the assumption of shared cultural knowledge to be met, according to the conventions of CA, the variance accounted for by the first factor must be at least three times the amount accounted for by the second factor [16] [18]. Meeting this assumption allows the application of a Bayesian formula to these estimates (factor loadings) to determine the "culturally correct" response to each question (answer key)."

\section{Results}

\subsection{Sentence Completion by Card Sort}

Table 1 shows the CA results for the data produced by the sentence completion/card sort task. The eigen-value ratio of the first two factors is 3.52, indicating that the assumption of shared cultural knowledge has been met. The cultural knowledge scores range from 0.23 to 0.93 with a mean of 0.615 (S.D. $=0.198$ ). With this sample size $(\mathrm{N}=28), 99 \%$ of their answers can be said to be "culturally correct" with a 0.99 confidence level (p-value $\leq$ $0.001)$.

Analysis of variance was used to determine whether level of cultural knowledge about HBP/HTN varied significantly by self-reported familiarity with the condition. The CA-produced knowledge scores for the 14 participants who had been diagnosed with HTN were not significantly different from the scores of the 11 who knew someone who had it and the 3 who felt they were "adequately" informed about it.

According to the answer key in Table 1, stress is implicated in the etiology of HBP/HTN, along with improper diet, lack of exercise/being overweight, drinking and/or smoking, heredity and aging. The results for the sentences about stress, diet, exercise/weight, substance use and heredity are in keeping with the a priori hypotheses. The recognition of aging as a causal/exacerbating factor in the shared EM is surprising, as this was not mentioned in the literature (or the narratives). Weather and contagion, as hypothesized, are confirmed not to be parts of the shared EM. Regarding treatments, the consensus is that medical doctors can help with HBP/HBP, but home remedies cannot. The consensus regarding consequences is that HBP/HTN is serious and life threatening, as hypothesized, and disabling, in that it changes a person's life once they have it. Also, as hypothesized, there is consensus that HBP/HTN is not painful, although some reference to dizziness, headache and other, specific pain did appear in the narratives. 


\subsection{Categories Coded from the Free Lists}

Table 2 shows the frequencies coded from the interviews of the 19 participants who listed HBP/HTN from memory. The same themes—stress, diet, physical activity and smoking — appear as causes (etiology), as well as preventions, and in many cases, treatments through their reduction or absence.

People talked about how the stresses of daily life are implicated in the etiology and onset of HBP or HTN (53\%). It could be chronic, ongoing stresses or a discontinuous event like the serious illness or loss of a loved one. Poor diet (42\%), and working too hard or too much (32\%) also appeared on many of the lists. Smoking was thought to be generally bad for people (16\%) and heredity was thought to be an unavoidable risk factor (16\%) for HBP/HTN.

The best way to prevent it was to avoid its causes. People did not think stress was easy to avoid, but $26 \%$ said it was a good idea to try. Most of the discussion revolved around improvements in diet (53\%), because diet is perceived to be something an individual can control. Twenty-one percent did mention that exercise, even walking to the store, would help with general health and therefore might help prevent HBP/HTN, while $11 \%$ recommended never starting to smoke or stopping if one had already started.

Almost everyone (95\%) said that once diagnosed, the best way to treat HTN would be to take the medications prescribed by their doctor. But they also mentioned that a healthier diet (42\%) and more exercise (21\%) would most likely help. How they approached treatment often depended on whether they saw HTN as a chronic condition or an acute one.

Only 26\% mentioned that HTN has no symptoms, while 21\% mentioned dizziness, and $16 \%$ each mentioned headache and another, specified pain as symptoms. What does not come out in this coding is that some people who said they perceived symptoms also said that they could tell when their pressure was up, and that they could modify their medication intake accordingly.

\subsection{Theme Analysis from Narratives}

The following sections give examples of the categories/model elements listed above, quoted directly from the narratives. The material is divided into Causes/Prevention (Stress, Diet, Exercise, Smoking, Heredity and Aging), and Treatments, Consequences and Symptoms.

\subsubsection{Causes/Prevention}

High blood pressure or hypertension is stress-related. Participants often mentioned stress or tension as a major cause of HBP/HTN. One man described the causal relationship as follows:

- ... I think the tension is what causes the hypertension. Well, it's part of the word. So, if you become tense enough, long enough, you become hyper! (MA82)

Hypertension-causing stress was associated with the challenges and aggravations of life, such as illness, work, and interpersonal relations. Several individuals spoke of discrete, traumatic events that might have or did result in an elevation of blood pressure.

- My blood pressure was ok, like 140. And now I go in and after all this stress I've had, you know, about my husband's illness and all that, you know. It's kind of, maybe that's what gotten me down. ... A lot of stress and something that you just can't do anything about it. (FMx73)

- Any time I'm going through anything traumatic, my blood pressure would shoot up... Like, if I'd go in and the doctor would say, "What's going on in your life, your blood pressure's up?” (FIr74)

Some felt that daily, chronic stress could ultimately elevate blood pressure.

- I've always had high blood pressure and I figured that was because my working, you know, you get all tensed up working... Tensed up, yeah. Lots of stress, aggravation. (FA80)

- You become tense, anxious, driving, you know the job is driving you, the family is driving you, the man or woman you're supposedly in love with isn't "doing right by you." And you become very tense and if you're not careful, then you get to feeling sorry for yourself, and that's when the hyper starts to set in. (MA82)

Others described blood pressure as going up and down in response to immediate, periodic life stresses.

- But you can have different types of high blood pressure. Mine was the type that comes and goes. Things that bother you, that cause it, you know. You have something comes up that bothers you... an incident that happened. And, maybe fear of something that you might have a sickness or something - the fear of that or what might happen could cause that, too. (FGr85) 
- You could have a normal blood pressure and be fine and go drive to the doctor's office and your blood pressure could be sky high, just from driving... tension or something. You've got to keep your eyes open or somebody isn't going fast enough and a lot of times somebody honks a horn at you-you did something wrong. (MAu77)

When discussing prevention or management of HBP in relation to stress, participants varied depending on whether they believed an individual could control stress.

- I don't think you can prevent hypertension. Because 9 out of 10 people, they have stress. They don't know where it comes from: an aggravation. (FA80)

- Try not to get nervous, I guess is the only thing I know. (MIt76)

- Well like I say, try to control your temper, which would be very easy. Just control your temper and try to stay cool. That's all I can say. (MAu77)

High blood pressure or hypertension is diet-related. Diet was cited by participants as a major cause of HTN, particularly a diet including red meat, fat, salt, spicy foods and sweets. Some felt that a combination of these would cause HBP.

- My husband didn't watch his diet. Anything that he wanted, I tell you when I married him, he ate a lot of foods that I thought was... I thought, this was just terrible. And he would ask me to cook it for him. And I said, “I wasn't brought up on that kind.” I mean, he had to have bacon in the morning and he would really fry about half a pound of bacon. And you know the doctors don't want you to eat too many eggs? He would put about three or four eggs on his plate. He was a big man and he had a big appetite. So, I think all the harm, he really did to himself, and it was with foods. (FB82)

- My brother's wife was ah, she ate all the wrong foods. She was born in Mississippi and brought up in Kentucky. Everything she ate, everything she cooked was fried in lard. And everything was fried. And she ate all the wrong foods. She didn't eat green vegetables. And the doctor said her blood pressure was way high. The doctor said, "If you don't stop this, you're going to have a heart attack and die." And she said, "If I do, I'll die happy.” And she never changed her diet and she did die from having a heart attack. Well, she always said, "I'm just not going to change. I'm going to eat what I like and if I die too quick, I'll die too quick." (MA81)

- I think the same thing causes high blood pressure that causes everything else. Eating spicy foods, eating a lot of red meat, eating sweets... (MIr79)

- Salt in the diet is very bad. Too much salt... closes the arteries... (FJ79)

- I can tell you what will give the high blood pressure to you: pork meat. She [his wife] don't need any pork anymore. She was born in a farm back in Kentucky and her dad kept pork hanging in the, you know, salt pork, how they used to hang it up in the barn and put salt all over it and all that mess? And then they eat that stuff it will give you the high blood pressure. Salt pork. She hasn't had any of that for years now. But she still suffer from it. I guess once you get it, you just don't get rid of it, right? But that will sure give it to you. [Do you have high blood pressure?] No, I never ate pork. Never did eat pork. My dad was a vegetarian and I'm a vegetarian today. [And you're pretty sure it comes from eating pork?] Yeah, that's one of the reasons. (MB75)

Participants also saw modifying diet as one of the ways to treat or manage high blood pressure.

- You don't eat a lot of meat, beef. You eat chicken and fish. What else? You don't eat a lot of sweets either. (FGr85)

- [Do you do something to get it down?] Diet plays a big part. You watch your diet-too much fat and too much this. [Anything else with the diet you need to watch in order to control your blood pressure?] Well, just grease and fatty foods. That's about all. I don't have to eat too many vegetables. I don't like salads. But that may have something to do with it. I should eat more vegetables, but I do eat fruit and that seems to help. (MJ81)

As in their discussion of diet-related management of high blood pressure, participants recommended avoiding certain kinds of food and eating healthier foods as an important way to prevent HBP in the first place.

- Eat the right foods, so you don't have all that fried food and high blood pressure. Most of what I eat I don't really like. But I eat it because it's good for me. (MA81)

- Your diet is a big thing. Keep your cholesterol down - that would be not many eggs or cheese. The right kind of cheese you can have. That would be a big item. (FGr85)

Participants also discussed diet in close conjunction with weight, and with exercise. Diet was seen as a means of manipulating one's weight, when this was perceived as a risk factor for developing HBP. 
- ... If you're too heavy, you have the liability of having high blood pressure. I think you should be lean, or they say anyhow. (MAu77)

- I've been able to keep my weight down. I was a little over-weight, but since I've been strict with staying on my diet, calories wise, been able to keep my schoolboy figure. Say that to people all the time. Our folks love that Southern cooking, and that's the worst thing for them. They can't control their weight. Usually, most of them great big people- three, four hundred pounds. All eat that old salt pork. (MB77)

High blood pressure or hypertension is related to exercise and weight. Exercise was linked with diet and weight in discussing the management or prevention of HBP.

- Change their diet and exercise. That's what everybody is supposed to do when they have high blood pressure. (MIr79)

- Diet is very important and our mode of life, also. [What do you mean by that?] Walk up the steps every once in a while. If you got to go down to the corner, instead of taking the car, walk it. (FJ79)

- Well, for one thing you watch your diet and you exercise. I walk three times a week down at the pier, I mean, down at the ocean on the strand. (FGr85)

One cited exercise as a means of managing HTN without linking it closely with diet:

- I know exercise is supposed to help. It's supposed to reduce high blood pressure... (FJ75)

High blood pressure or hypertension is related to drinking or smoking. Smoking was also identified as a cause of HBP, by itself or in combination with other risk factors such as drinking or overexertion, or with being overweight, all of which are considered to be under the personal control of an individual.

Smoking was seen as a harmful behavior that increases the risk of developing HBP/HTN and exacerbates it once it has developed.

- Don’t smoke-I ain't smoked for thirty years or something like that. (MA93)

- Just like my doctor says..., "Quit smoking and lose weight.” (MIr79)

A woman whose husband used to smoke and had high blood pressure said,

- Well, he [the doctor] took the cigarettes away from him [her husband]. (FB82)

Blood pressure is perceived to rise and fall in response to how individuals behave with regard to diet, physical activity, weight control and smoking, all of which involve individual behaviors that can be modified. Thus individuals are seen to be capable of - and personally responsible for-changing these risk factors and, through them, their blood pressure.

High blood pressure or hypertension is hereditary; it runs in families. On the other hand, hereditary is something that cannot be controlled by individuals.

Of her husband, one woman said,

- And of course I think that high blood pressure ran in the family, so by then both had problems. But, he looked. He was an old man. He was 79. (FB82)

When reminded that he had mentioned that his mother's family had a history of stroke, and asked whether there was anything he had learned to watch out for, one man said,

- Well, high blood pressure, obviously, is the first thing. (MA81)

High blood pressure or hypertension is age-related. Only one person mentioned that HTN gets worse with age. He also saw age as making it more difficult to cope with, but he never said it was caused by aging. In discussing his wife's experience, he said:

- She [my wife] had blood pressure all her life, ever since she was a young girl. But, you know, as you get older, it gets a little more severe. When she was young she was able to cope with it, you know. Now, at her age, it's hard to cope with. (MIt76)

In contrast, a woman saw increasing age as helping her to manage the stresses in life:

- I think I've had a lot of experience, and maybe I call that growing old. You know, more ah, take things in stride. I don't make too much of anything. (FMx73)

\subsubsection{Treatments}

A doctor can help with high blood pressure or hypertension. Almost all participants agreed that medication prescribed by a doctor is an effective treatment for HTN.

- ... if it gets too bad, I go to the doctor. I make an appointment. I go get in as fast as I can. (FA80)

- ... as soon as I had this report of going over 90, I went to the doctor immediately and he gave me some pills to take. Knocked it down. (MA81) 
Medication prescribed by a doctor was mentioned as both a treatment and a way to prevent hypertension.

- [How do people prevent hypertension?] Go to the doctor and take the medication to lower the blood pressure. (MA82)

- Well most times, they're on medication to treat high blood pressure. It is medication from the doctor. I don't know of any medication you could buy from the drugstore. (FB77)

- [What about your wife?]... Well, the doctors they're giving her medication for her hypertension to drop the high part of it back to normal. So by going periodically to her doctor she keeps it under control. When she sees it getting out of control, she takes the medication to keep it down. (MB75)

Some also mentioned the need to take prescribed medication for HTN on a regular basis.

- Take your pills regularly. You got to take all that stuff regularly or you're gonna fall down. (MMx74)

- I take medication for high blood pressure. I take it every day. Here's all my medication-all on one page. I take all of those, every day. I'm under doctor's care all the time. (MAu77)

Others did not take their hypertension medication regularly, although they knew they should:

- There's pills that you can take to prevent hypertension. The doctor gave me a prescription for pills. I don't know the name of them even. I've got them in the drawer. I don't take them too often. I should, but I don't, because I can feel when my blood pressure is normal. I can almost feel it. (MJ81)

- I wish there was a natural way to prevent [high blood pressure], where you didn't have to take pills. I've tried a few things, [they] don't work. (MMx76)

Yet two women spoke about successfully avoiding prescription medication by using alternative approaches.

- Things are going out into the world about how you can lower your blood pressure. You don't have to take medication. There's certain things you can do. Watch your fat. (FA79)

- I was taking high blood pressure pills but I take herbs now and my pressure's normal with the herbs. Since I was on the herbs, I've been off my blood pressure pills for over a year and the doctor says that I'm just excellent. (FIr74)

\subsubsection{Consequences}

High blood pressure or hypertension is serious, life threatening, and disabling. Participants described hypertension as a condition with potentially serious consequences, including stroke, heart attack, death and permanent disability.

- Well, I guess if you have high blood pressure you are prone to or possible candidate for stroke. So, hypertension is a killer. There's no doubt about it. It may come out as a stroke, high blood pressure, heart attack (MA82)

- [What causes heart problems?] Well, I think high blood pressure would be one of your starts. (MMx74)

- I know excessive high blood pressure will give you stroke. (MJ81) Several talked explicitly about the seriously disabling consequences of hypertension-caused stroke.

- Every case of these people, they all have high blood pressure. That's what caused the stroke. In those days, they called it paralytic stroke because it paralyzed them. (MA81)

- Well I think [blood pressure] could cause a stroke if it gets real high... And they get paralyzed like, part of their body, whatever has the stroke gets paralyzed: Temporarily, maybe sometimes permanently. (MIt76)

\subsubsection{Symptoms}

When asked how they could tell if they had HBP, some participants said it requires a test.

- Oh, the only way I know is doctor takes your blood pressure and it’s high. (FJ75)

- I have absolutely no symptoms except it shows up on the blood pressure test. (FJ79) But others were sure they could tell from symptoms.

- When I have it, I used to get pressure headaches. Really, pressure headaches. And sometimes I'd feel kind of flushed. Once in a while I'd bend over and I'd be kind of dizzy. (FA80)

- Let's see. You get this ache in the back of your neck. And then you feel sort of dizzy. And ah, of course you get this little thing on your chest. I wouldn't say it's a pain, but sort of like a heavy something on your chest. (FMx73)

One woman had heard of perceptible symptoms, but she herself never experienced them.

- They may be dizzy at times. And ah, I didn’t have that symptom, though. In fact, I didn’t know I had high blood pressure. (FGr85) 


\section{Discussion}

The older adults interviewed in Los Angeles for this study, some of whom had been diagnosed with HTN and some of whom had not, share an EM of HBP/HTN that has elements in common with the EM of ethnically and nationally diverse patients interviewed for other recent studies [19]-[23]. The persistence and geographic distribution of such lay or popular models further underscores their relevance.

They identified stress as a major cause or risk factor, as had the informants interviewed by Blumhagen [5] [6], Heurtin-Roberts [8], and Garro [7]. Five recent studies that used Kleinman's EM framework to interview HTN patients also identified stress as a perceived causal/risk factor in this disease. Mexican American adults in Texas [19], Filipino American adults in Los Angeles [20], African American, Hispanic and White veterans in the "Northeast" and "Southwest” of the United States [21], Nigerian hospital patients [22], and Ghanaian, AfricanSurinamese, and Dutch patients in Holland [23] all include stress in their etiological explanations.

Lifestyle factors also are implicated in the etiology of HTN/HBP. The EM data collected in Los Angeles show the strong influence of a public health education emphasis on adopting a lifestyle that generally promotes good health and specifically includes eating healthy foods, exercising properly, avoiding substance abuse, maintaining optimal ideal weight for height and getting adequate rest. Poor dietary practices and lack of exercise were mentioned as problems leading to HTN in all the recent studies listed above. Overindulgence in alcohol was mentioned by Filipino Americans [20] and Nigerians [22], and being overweight was mentioned in the Dutch study [23]. Alcohol abuse and being overweight also are causes shared with Blumhagen's [5] [6] Seattle veterans, Garro’s [7] Canadian Ojibway and Heurtin-Roberts' [8] African Americans (when discussing "high blood”) as well. In addition, Garro’s informants shared a concern about overexertion and Blumhagen’s informants shared a concern about smoking with the older adults in Los Angeles.

The nature of the relationship between lifestyle and HBP was explained in the narratives. The model involves moderation, in that too much of one thing (salt, fat, alcohol) or too little of another (exercise, rest) are seen to cause harmful imbalance. These comments reflect the public health education emphasis on personal responsibility for health, and, by logical extension, personal blame for illness. To the extent that stressful situations, response and coping are under the control of an individual, stress too can be seen as part of a lifestyle choice that falls under personal control.

However, some causal factors are understood to be completely beyond an individual's control. The Los Angles participants recognized heredity as an alternative explanation for HTN, as did the participants in the 3 studies that preceded this one, as well as the Filipino Americans in Los Angeles [20] and Mexican Americans in Texas [19]. It is worth noting that heredity was rarely mentioned when participants were asked to list causes of HBP from memory during the free list procedure. However, it was mentioned spontaneously during discussions of other illnesses, and once asked in the card sort/sentence completion procedure, was endorsed as a causal factor.

Aging, also outside of an individual's control, was not mentioned in any of the 5 recently published studies as an etiological factor in HTN/HBP. In the narratives, it was mentioned only in reference to enhancing the ability to cope with HTN or with stress in general in later life. Nonetheless, when offered the opportunity, the participants endorsed aging as part of their EM for HTN/HBP.

In their narratives, older adults in Los Angeles identified perceptible symptoms of HBP/HTN, including headaches, dizziness and tiredness, as did the veterans in Seattle [5] [6], the Ojibway in Canada [7], the Filipino Americans in Los Angeles [20], the Dutch, Ghanaians and Surinamese in Holland [23], and the American veterans [21]. The only symptom-related sentence frame question subjected to consensus analysis was whether HBP/HTN was painful or led to discomfort. The consensus was that it did not. People who considered HBP to have perceptible symptoms were likely to think it should be treated only when symptoms were present, while those who thought HBP was always there "silently," tended to think it should be treated regularly over time even in the absence of perceptible symptoms.

Judging by their narratives, most participants viewed this illness as having flare-ups. They felt that, after the onset, treatment is synonymous with prevention of the next flare-up (or worse). Most preventive interventions, not including medication, in turn, involved the removal of causes, such as the lifestyle "violations" mentioned above. Thus the endorsements of the sentences regarding the exacerbating effects of poor diet, overweight and lack of exercise, and substance abuse, could tentatively be taken as endorsements of the preventive effects of improving one's lifestyle. 
Also similar to participants in most other studies, the Medicare-eligible older adults in Los Angeles saw HTN as a condition with potentially serious health consequences. They agreed that it was life threatening and disabling. In the narratives they talked about stroke, paralysis and eventually death as outcomes of the condition, in keeping with the medical model and the messages of public health education.

Similarities across studies conducted with informants of different ages and ethnicities, and in many different locations, suggest that certain perceptions of HTN are widely shared. Such widespread similarity is likely due, at least in part, to interaction between popular ideas and professional biomedical and public health information. That is, lay people create aggregate models out of their own experience, the experience of family, friends and neighbors, information in the media, and knowledge gained during their consultations with health professionals. Many of the diagnosed participants whose narratives are quoted above made explicit references to what their doctor told them about the nature, causes and treatment of HTN.

But the transmission of information from professional to lay person is far from perfect. While it is likely that the consensus that HTN is not painful and does not result in discomfort comes from biomedical understandings that HBP is a "silent killer," it is also true that some simultaneously hold contradictory ideas about perceptible symptoms.

Narrative comments, although not part of the cultural consensus, identify several areas of potential concern for both clinicians and public health professionals. For example, some participants believed that perceptible symptoms accompany HBP and that as blood pressure rises and falls episodically, it is possible to tell from symptoms when blood pressure is high. This suggests that greater educational emphasis needs to be placed on the importance of regularly taking medication and routinely checking blood pressure even in the absence of symptoms.

In summary, Consensus Analysis (CA) permits a confident statement that there are shared cultural understandings in this group of older adults in Los Angeles about the nature, causes/prevention and treatment of HBP/ HTN; narrative analysis enhances the findings by adding more explanatory detail. Limiting the card sort/ sentence completion procedure to elements that are relevant across many illnesses allows systematic comparison across illnesses and populations. However, disease-specific elements (e.g. the significance of salt in HTN) are lost in this procedure. One solution might be to develop a list of core sentences for use in research across illnesses or diseases, and other, more illness-specific sentences to be added to the core when one specific disease/illness is of interest.

\section{Conclusion}

New advances in the understanding of HTN become available to biomedical practitioners, clinically diagnosed patients and the general population on a regular basis; however, the flow of information is unreliable. As an example, it has been medically recognized for nearly two decades [24] that dietary changes and subsequent weight loss can reduce or even eliminate a patient's need for medication, provided they remain under the care of a physician. While it is likely that physicians who treat HTN are aware of this study by now, and may have passed the knowledge on to their clinically diagnosed patients, it seems less likely that this has become "common knowledge" for the general public. This suggests that periodic studies of shared EM of HTN/HBP using consensus analysis to assess what information has been incorporated into the popular culture at any given time could be useful in the design of public health education and interventions.

\section{Acknowledgements}

The research presented in this paper was supported in part by a NIH-NIA FIRST Award, "Decisions about HMO Use for Late Life Illness" granted to MLH. We also would like to thank the many undergraduate and graduate student assistants who helped with this project.

\section{References}

[1] Kleinman, A. (1977) Concepts and a Model for the Comparison of Medical Systems as Cultural Systems. Social Science and Medicine, 12, 85-94.

[2] Kleinman, A. (1980) Patients and Healers in the Context of Culture. University of California Press, Berkeley.

[3] Good, B. (1977) The Heart of What's the Matter: The Semantics of Illness in Iran. Culture, Medicine and Psychiatry, 2 , 
107-138. http://dx.doi.org/10.1007/bf00114809

[4] Helman, C.G. (1978) "Feed a Cold, Starve a Fever"-Folk Models of Infection in an English Suburban Community and Their Relation to Medical Treatment. Culture, Medicine and Psychiatry, 2, 107-138. http://dx.doi.org/10.1007/BF00054580

[5] Blumhagen, D. (1980) Hyper-Tension: A Folk Illness with a Medical Name. Culture, Medicine and Psychiatry, 4, 197227. http://dx.doi.org/10.1007/BF00048414

[6] Blumhagan, D. (1982) The Meaning of Hypertension. In: Chrisman, N.J. and Maretzki, T.W., Eds., Clinically Applied Anthropology, D. Reidel, Dordrecht, Holland, 297-323.

[7] Garro, L. (1988) Explaining High Blood Pressure: Variation in Knowledge about Illness. American Ethnologist, 90, 98-119. http://dx.doi.org/10.1525/ae.1988.15.1.02a00070

[8] Heurtin-Roberts, S. (1993) "High-Pertension”-The Uses of Chronic Folk Illness for Personal Adaptation. Social Science and Medicine, 37, 285-294. http://dx.doi.org/10.1016/0277-9536(93)90260-B

[9] Baer, R., et al. (1999) Cross-Cultural Perspectives on the Common Cold: Data from Five Populations. Human Organization, 58, 251-260. http://dx.doi.org/10.17730/humo.58.3.n4413t15t4220567

[10] Baer, R., et al. (2008) Cross-Cultural Perspectives on Physician and Lay Models of the Common Cold. MAQ, 22, 148-166. http://dx.doi.org/10.1111/j.1548-1387.2008.00012.x

[11] Schrauf, R.W. and Iris, M. (2011) Using Consensus Analysis to Investigate Cultural Models of Alzheimer's Disease. In: Kronenfeld, D.B., Bennardo, G., de Munck, V.C. and Fischer, M.D., Eds., A Companion to Cognitive Anthropology, Blackwell Publishing Limited, Oxford, 548-568. http://dx.doi.org/10.1002/9781444394931.ch29

[12] Hurwicz, M. and Rose, M. (2015) Older Adults’ Explanatory Models of Colds and Flu. Health, 7, 1183-1195. http://dx.doi.org/10.4236/health.2015.79133

[13] Helman, C.G. (1994) Culture, Health and Illness: An Introduction for Health Professionals. 3rd Edition, ButterworthHeinemann Ltd., Oxford.

[14] Hurwicz, M. (2008) From Free List to Consensus: Explanatory Models of Late Life Illness. Paper Presented to the American Anthropological Association, San Francisco. (Unpublished)

[15] Borgatti, S.P. (1992) ANTHROPAC 4.0. Analytic Technologies, Columbia.

[16] Weller, S.C. (2007) Cultural Consensus Theory: Applications and Frequently Asked Questions. Field Methods, 19, 339-368. http://dx.doi.org/10.1177/1525822X07303502

[17] Romney, E.K., Weller, S. and Batchelder, W.H. (1986) Culture as Consensus: A Theory of Culture and Informant Accuracy. American Anthropologist, 88, 313-338. http://dx.doi.org/10.1525/aa.1986.88.2.02a00020

[18] Weller, S.C. and Romney, A.K. (1988) Systematic Data Collection. Newbury Park.

[19] Kolb, S.E., et al. (2011) Perceptions about High Blood Pressure among Mexican American Adults Diagnosed with Hypertension. Family \& Community Health, 34, 17-27. http://dx.doi.org/10.1097/FCH.0b013e3181fdec84

[20] Dela Cruz, F.A. and Galang, C.B. (2008) The Illness Beliefs, Perceptions, and Practices of Filipino Americans with Hypertension. Journal of the American Academy of Nurse Practitioners, 20, 118-127. http://dx.doi.org/10.1111/j.1745-7599.2007.00301.x

[21] Bokhour, B.G., et al. (2012) The Role of Patients' Explanatory Models and Daily-Lived Experience in Hypertension Self-Management. Journal of General Internal Medicine, 27, 1626-1634. http://dx.doi.org/10.1007/s11606-012-2141-2

[22] Taylor, K.D., et al. (2012) Explanatory Models of Hypertension among Nigerian Patients at a University Teaching Hospital. Ethnicity \& Health, 17, 615-629. http://dx.doi.org/10.1080/13557858.2013.771151

[23] Beune, E.E.A.J., et al. (2006) “Under Pressure”: How Ghanaian, African-Surinamese and Dutch Patients Explain Hypertension. Journal of Human Hypertension, 20, 946-955. http://dx.doi.org/10.1038/sj.jhh.1002094

[24] National Institutes of Health (1998) Lifestyle Changes Reduce Need for Blood Pressure Drugs in Elderly. Press Release. March 17, 1998. 